\title{
Should it stay or should it go?
}

\author{
Nicholas G. Smedira, MD

\footnotetext{
From the Department of Thoracic Surgery, The Cleveland Clinic Foundation, Cleveland, Ohio; and the Department of Cardiovascular Surgery, The Cleveland Clinic Foundation, Cleveland, Ohio. Disclosures: Author has nothing to disclose with regard to commercial support.

Received for publication Sept 8, 2015; accepted for publication Sept 9, 2015.

Address for reprints: Nicholas G. Smedira, MD, Department of Thoracic/Cardiovascular Surgery, The Cleveland

Clinic Foundation, 9500 Euclid Ave, Desk F25, Cleveland, OH 44195 (E-mail: smedirn@ccf.org). J Thorac Cardiovasc Surg 2016;151:199-200

$0022-5223 / \$ 36.00$

Copyright (C 2016 by The American Association for Thoracic Surgery

http://dx.doi.org/10.1016/j.jtcvs.2015.09.038
}

In their 1982 hit, "Should I Stay or Should I Go?" from the album Combat Rock, the punk rock band The Clash sing of a tumultuous relationship and say if you are good to me, "I will be there till the end of time." In their article in this issue of the Journal, Baldwin and colleagues suggest that once the pump has nicely done its job of recovering the failing ventricle, permanently leaving an entire continuous-flow left ventricular assist device in situ may be the safest and least morbid approach to transitioning to device independence (Figure 1).

Baldwin and colleagues note that Dr DeBakey's first patient requiring temporary left ventricular support had both the inflow and outflow grafts ligated without sequelae. The major difference with the patients reported in their article is the presence of a large retained intravascular and intracardiac metal inflow cannula. Combat and urban penetrating trauma studies have shown that retained objects that are thought to be sterile do not require removal unless they are at risk for embolization to the lungs or extracardiac organs.

There are good reasons to avoid unnecessary trauma when removing retained objects. This principle was unknown when President James A. Garfield was shot while awaiting a train on July 2, 1881. The first wound was superficial, but the second bullet entered the right flank, traversed the first lumber vertebra, and settled harmlessly in the left retroperitoneal space. At that time it was thought that (1) the bullet must be removed and (2) the patient should not take any food by mouth. Garfield's doctors repeatedly explored the right flank wound with unsterile fingers and instruments, leading to a wound infection, sepsis, and death a few months later. Malnutrition didn't help. President Garfield lost 100 pounds on a rectal diet consisting of a cocktail of beef bouillon, egg yolks, milk, whiskey, and opium drops. ${ }^{1}$

Balancing the risks of a complex operation to remove the device and a much simpler operation leaving a large volume of material in place with a large intravascular object can be difficult. In this very small study of only 27 patients with 4 different interventions, we have some new information to guide us, but the decision remains challenging. Nearly

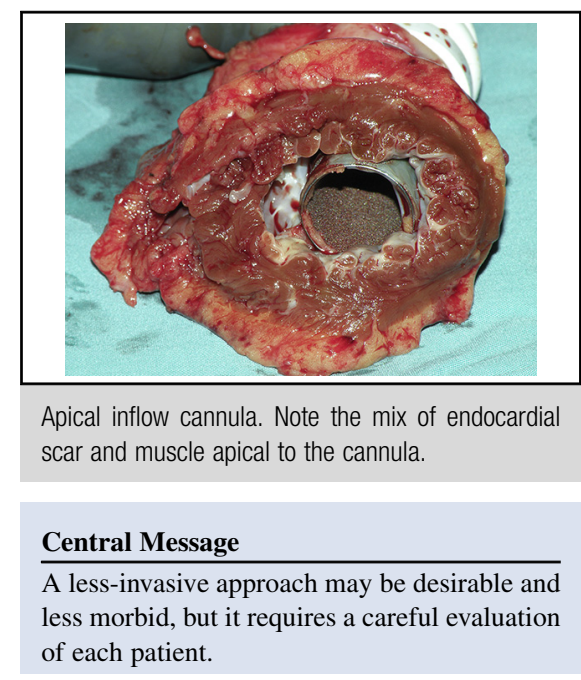

See Article page 192.

See Editorials page 10 and 13.

one-third of the patients required reoperation. It appears that we can conclude that patients with infected pumps need as complete a removal of infected material as is safely possible. This follows the principles developed for the treatment of prosthetic valve endocarditis and total joint infections. Whether small remnants of retained material at the left ventricular apex or ascending aorta need vascularized flap coverage and obliteration of all dead space is unknown, but this is probably a prudent approach because infection-related disruptions can be lethal.

For uninfected pumps, the main question remains whether the retained inflow cannula poses a long-term risk to the patient. Baldwin and colleagues conclude that it does not; however, only 14 patients had a retained left ventricular cannula. Of the 4 strokes in the study, 3 occurred in patients with a retained cannula; however, the strokes appear to have been related to the outflow graft management rather than to the inflow cannula. For most centers, device intervention will be prompted by pump malfunction or partial or complete thrombosis, rather than ventricular recovery. Management of the outflow graft to avoid embolization of thrombus becomes critical. Computed tomographic imaging can be helpful to assess outflow graft thrombus. but in my experience the diagnostic accuracy is low, with frequent false-positive and false-negative results. We have tried a combined subcostal incision for the pump exchange and a left second interspace incision to gain direct control of the aorta beyond the anastomosis, but this is 


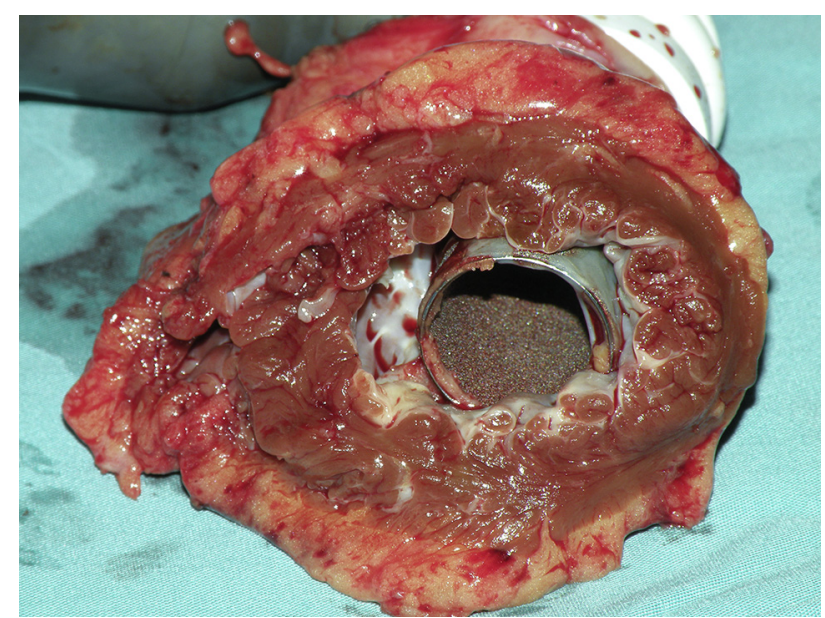

FIGURE 1. Apical inflow cannula. Note the mix of endocardial scar and muscle apical to the cannula.

easier said than done. It may be that if there is any doubt about outflow graft thrombus, then treating the outflow graft like a diseased saphenous vein graft with a no-touch technique with control of the anastomotic site may be the safest approach.

Another concern not mentioned by Baldwin and colleagues is the arrhythmogenic potential of the inflow cannula and the myocardium around the cannula. One patient died of ventricular arrhythmias at 1.5 years after explantation, and inspection of the Kaplan-Meier survival curves suggests that this occurred in a patient with a retained inflow cannula. The recovered ventricles were small, measuring $4.9 \mathrm{~cm}$, and it is well known that endocardial contact with the cannula during pump support can generate arrhythmias. In addition, we found that in $14 \%$ of the patients with left ventricular assist device support requiring ablation for recurrent ventricular tachycardia, the muscle just beyond the apical sewing ring was the source of the ventricular tachycardia. ${ }^{2}$ If not present, an implantable cardioverter defibrillator may be an important component of care for all patients with an apical cannula.

For decades, the surgeons at the Texas Heart Institute have been pioneers in developing mechanical pump technology and the surgical techniques for their insertion. Their next frontier is device removal. A less-invasive approach may be desirable and less morbid, but it requires a careful evaluation of each patient. Only then can we determine whether the pump or its components can stay or should go.

\section{References}

1. Schaffer A. A president felled by an assassin and 1880's medical care. The New York Times. July 25, 2006. Available at: http://www.nytimes.com/2006/07/25/ health/25garf.html?pagewanted=all\&_r=0. Accessed August 30, 2015.

2. Cantillon DJ, Bianco C, Wazni OM, Kanj M, Smedira N, Wilkoff B, et al. Electrophysiologic characteristics and catheter ablation of ventricular tachyarrhythmias among patients with heart failure on ventricular assist device support. Heart Rhythm. 2013;9:859-64. 